\title{
Cytoprotection Against Oxidative Damage by Nrf2-regulated Genes
}

\author{
Mi-Kyoung Kwak ${ }^{1}$ and Thomas W. Kensler ${ }^{2}$ \\ ${ }^{1}$ College of Pharmacy, Yeungnam University, Gyeongsangbuk-do 712-749, Korea \\ ${ }^{2}$ Department of Environmental Health Sciences, \\ Johns Hopkins University Bloomberg School of Public Health, Baltimore, MD, USA
}

Received August 28, 2007; Accepted September 11, 2007

\begin{abstract}
Chronic oxidative stress produced by exposure to environmental chemicals or pathophysiological states can lead animals to aging, carcinogenesis and degenerative diseases. Indirect antioxidative mechanisms, in which natural or synthetic agents are used to coordinately induce the expression of cellular antioxidant capacity, have been shown to protect cells and organisms from oxidative damages. Electrophile and free radical detoxifying enzymes, which were originally identified as the products of genes induced by cancer chemopreventive agents, are members of this protective system. The NFE2 family transcription factor $\mathrm{Nr} 2$ was found to govern expression of these detoxifying enzymes, and screening for Nrf2-regulated genes has identified many gene categories involved in maintaining cellular redox potential and protection from oxidative damage as Nif2 downstream genes. Further, studies using nnf2-deficient mice revealed that these mutant mice showed more susceptible phenotypes towards exposure to environmental chemicals/carcinogens and in oxidative stress related disease models. With the finding that cancer chemopreventive efficacy of indirect antioxidants (enzyme inducers) is lost in the absence of Nrf2, a central role of $\mathrm{Nrf2}$ in the antioxidative protective system has been firmly established. Promising results from cancer prevention clinical trials using enzyme inducers propose that pharmacological interventions that modulate $\mathrm{Nrf2}$ can be an effective strategy to protect tissues from oxidative damage.
\end{abstract}

Key words: Oxidative damage, Indirect antioxidants, Enzyme inducers, Nrf2, Keap1.

\section{INDIRECT ANTIOXIDANTS}

It is recognized that oxidative stress is involved in the etiopathogenesis of a variety of human afflictions: aging, cancer, artherosclerosis, neurodegenerative diseases, and others, and there has been a long standing interest in the development of prophylactic measures to try to retard, block, or perhaps even reverse early and later stages in the progression of these different diseases. Traditionally, the approach has been to use the classical anti-oxidants - radical scavengers or interceptors that typically function as electron or $\mathrm{H}$-atom donors. However, there are some pharmacological barriers to this type of approach. Typically, these molecules have rather short pharmacodynamic half-lives unless they are recycled, such as the case with the vitamin $E$ molecule. We need relatively high dosage, frequent adminis-

Correspondence to: Mi-Kyoung Kwak, College of Pharmacy, Yeungnam University, 214-1 Dae-dong, Gyeongsan-si, Gyeongsangbuk-do 712-749, Korea

E-mail:mkwak@yumail.ac.kr tration; we need to get the anti-oxidants at the right place at the right time and for extended periods of time.

But there is another approach - using indirect antioxidants that can induce the expression of genes that scavenge, detoxify, or otherwise attenuate free radicals and their damage products. These molecules appear to have very protracted pharmacodynamic half-lives. Thus, they act for a long period of time. We need fairly low concentrations and we only need infrequent administration because of their protracted pharmacodynamic action. Some questions that will be addressed about these indirect anti-oxidants are, "What kind of chemicals, in fact, are they?" "What is the sensor for these indirect antioxidants?" "What genes are coordinately regulated through this sensor and how might they contribute to protection against a variety of oxidative stress mediated diseases?"

Paul Talalay and colleagues have now defined nine different chemical classes of indirect antioxidants (Talalay et al., 1988). These include isothiocyanates, which are constituents of a variety of different plants, dithiolth- 
iones, which are both natural and synthetic in origin, a variety of Michael reaction acceptors, arsenicals and heavy metals, hydroperoxides, vicinal dimercaptans, oxidized diphenols, phenylene diamines, quinones, as well as carotenoids. Overall, there is a wide diversity of agents that apparently do not share any common structural features. Some are oxidants, others are reductants and some appear to have no redox chemistry associated with them. Many of them are electrophiles; however, are a few nucleophiles, many are substrates for glutathione transferases, but the unifying feature appears to be that they are chemically reactive with sulfhydryl groups by oxidation or alkylation. This reactivity provides some key clues as to their molecular targets.

It has been our interests to identify both mechanisms and consequences of upregulation of genes involved in protection against chemical carcinogenesis using one of these indirect antioxidant classes of compounds, dithiolthiones (e.g., oltipraz and 1,2-dithiole-3-thione (D3T)). Using classical biochemical approaches as well as differential display, we recognized that there were several classes of genes that could be coordinately regulated, including those involved in carcinogen detoxication glutathione S-transferases (GSTs) and UDP-glucuronosyl transferases; among many, but also recognizing that there are a number of antioxidative genes that are coordinately up-regulated, including quinone reductase (NQO1), the heavy and light chains of ferritin, which are involved in iron sequestration, heavy and light chains of $\gamma$ glutamyl cysteine ligase (the rate limiting enzyme in the biosynthesis of glutathione (GSH)), manganese superoxide dismutase, and catalase (Kensler, 1997; Otieno et al., 2000; Primiano et al., 1996). Also a number of inducible genes that appear to be anti-inflammatory in their activities, including one that we originally cloned as dithiolthione inducible gene 1 , which now has a number of names including leukotriene B4 dehydrogenase or NADPH alkene/one oxidoreductase (Dick et al., 2001; Primiano et al., 1996; Primiano et al., 1998).

\section{THE NRF2/KEAP1 SIGNALING PATHWAY REGULATES THE ACTIONS OF INDIRECT ANTIOXIDANTS}

One feature that was common to all of these genes that we, and others in the field, were describing was that they appeared to share a common mode of regulation. The upstream sequences of these genes contained one or more copies of an "antioxidant response element" (ARE) with the core sequence of 5'-GTGAGnnnGCA-3' (Wasserman and Fahl, 1997). This finding provided some key clues as to how these enzymes were regulated. There continues to be a lot of activity to understand which transcription factors interact with the ARE to drive the tran-scriptional activation of these genes by these many different classes of indirect antioxidants. A number of laboratories identified the transcription factor $\mathrm{Nrf2}$ as a critical player in the upregulation of AREresponsive genes. Nrf2 is a CNC (cap'n'Collar) basic leucine zipper (b-ZIP) transcription factor. There are a number of other members of b-ZIP transcription factor family; however, Nrf2 appears to be the most important member of this family in terms of regulating gene expression through the ARE.

Itoh et al. (1997) showed that homozygous disruption of $n$ rf 2 in mice largely abrogated the inducible expression of GST and NQO1 by the phenolic antioxidant tertbutylhydroxyanisole $(t-\mathrm{BHA})$ in liver and intestine. Other studies demonstrated that $\mathrm{Nr} 2$ found to bind to the AREs of murine GSTA1, rat GSTA2, human NQO1, and human $\gamma$-glutamyl cysteine ligase genes (Itoh et al., 1997; Kang et al., 2000; Venugopal and Jaiswal, 1996; Wild et al., 1999). As shown in Fig. 1, it is now recognized that for the binding to the ARE, Nrf2 needs to form a dimer with other leucine zipper proteins. Small Maf proteins and AP-1 complex including MafK, JunD,

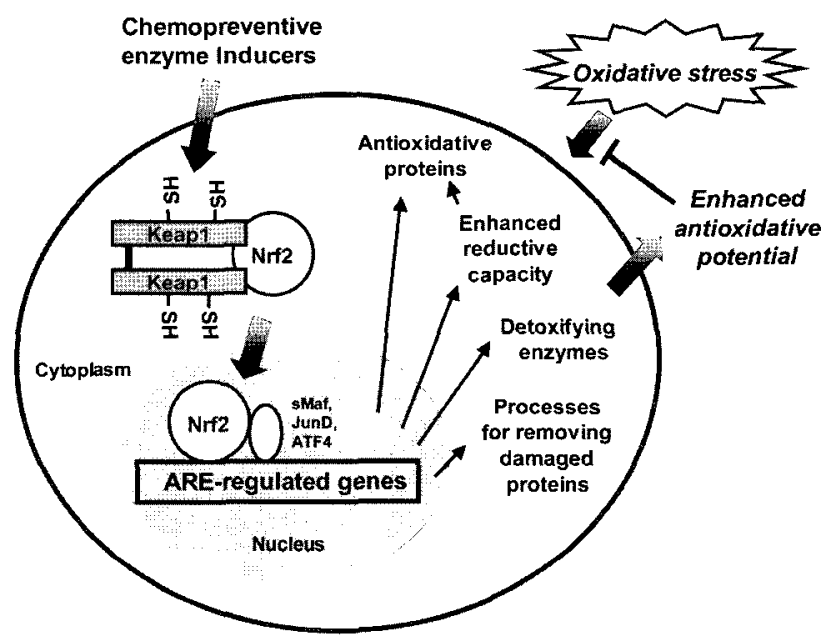

Fig. 1. Cytoprotective role of Nrf2 against oxidative stress. Chemopreventive enzyme inducers trigger the release of Nrf2 from a cytoplasmic inhibitor Keap 1 by altering the sulfhydryl bonds in Keap1 protein. Accumulated Nrf2 within nucleus transactivates the ARE-bearing genes and these genes involve in detoxification of environmental chemicals, enhancement of cellular reducing potential, and facilitation of the repair/removal of oxidatively damaged proteins. Collectively, these inducible genes by the keap1-Nrf2 pathway elevate cellular antioxidative potential and play an important role in cytoprotection against oxidative stress-associated tissue injuries. 
cJun, cFos and ATF4 have been recognized as partner proteins of Nrf2 (Itoh et al., 1997; Venugopal and Jaiswal, 1996, 1998; Wild et al., 1999). Further studies on the gene regulation by chemopreventive, indirect antioxidants in nrf2-disrupted mice have expanded the list of genes regulated by $\mathrm{Nrf2}$. It has been shown that nrf2-disrupted mice lost inducibility of GSTs (GSTA1-5, GSTM1/5 and GSTP1/2) and NQO1 in small intestine following treatment with indole-3-carbinol, $t$-BHA and ethoxyquin (McMahon et al., 2001). Also, inducible expression of GSTA1, NQO1, microsomal epoxide hydrolase, $\gamma$-glutamyl cysteine ligase, UDP-glucuronosyl transferase $1 \mathrm{~A} 6$ and $\mathrm{HO}-1$ by 1,2-dithiole-3-thione was largely attenuated in livers of nif2-disrupted mice (Kwak et al., $2001 b$ ). Abrogation of inducibility of antioxidative genes in nrf2-disrupted mice was also detected in mouse liver, lung, small intestine and forestomach following oltipraz treatment (Ramos-Gomez et al., 2003). Other antioxidative genes including peroxiredoxin MSP32 and cysteine membrane transporter A170 were identified as Nif2dependent genes in peritoneal macrophages (Ishii et al., 2000).

These results collectively support the essential role of $\mathrm{Nrf2}$ in the induction of multiple antioxidative genes. In addition, the cytoprotective role of antioxidative genes in chemical-induced carcinogenesis was further supported by the studies Ramos-Gomez et al. (2001) and Fahey et al. (2002). Pretreatment with oltipraz or sulforaphane significantly inhibited gastric tumor formation induced by benzo[a]pyrene in wild-type mice, but these agents did not show any protective effect in nrf2-disrupted mice (Ramos-Gomez et al., 2001).

\section{REGULATION OF NRF2}

The cytoskeletal-binding protein Keap1 plays a central role in the regulation of Nrf2 activity. Keap1 (Kelchlike ECH-associated protein1) was isolated as an inhibitor protein of Nrf2 by yeast two-hybrid screening (Itoh et al., 1999). Keap1, which associates with F-actin in cells, anchors Nrf2 in the cytoplasm through binding to the Neh2 domain of Nrf2. Targeted disruption of the keap1 gene in mice clearly demonstrated the crucial role of Keap1 in the regulation of Nrf2 (Wakabayashi et al., 2003). Levels of proteins for GSTs and NQO1 in livers of young keap1-disrupted mice were substantially higher than those of age-matched wild-type mice. Consistent with this observation, constitutive nuclear levels of Nrf2 and transcript levels for NQO1 in livers and fibroblasts from keap1-disrupted mice were high compared to tissues and cells from wild-type mice (Kwak et al., 2004; Wakabayashi et al., 2003). However, exact molecular mechanism of repressive role of Keap1 on Nrf2 is not fully understood at this time. Repressive role of Keap1 in Nrf2 function has been accounted for by two observations; i) Dissociation of Nrf2 protein from Keap1 can be triggered by treatment with sulfhydryl-reactive chemicals such as di- ethylmaleate (Itoh et al., 1999). A conformational switch through changes in sulfhydryl bonds of cysteine residues in Keap1 protein following treatment with chemicals has been hypothesized as a molecular mechanism of $\mathrm{Nrf2}$ activation. Wakabayashi et al. have demonstrated that $\mathrm{C} 273$ and C288 of Keap1 are the critical sensors modified by chemopreventive agents that lead to the disruption of the Keap1-Nrf2 complex (Wakabayashi et al., 2004). ii) Keap1 is also involved in facilitation of Nrf2 degradation by acting as an adaptor protein of E3 ubiquitin ligase (Kobayashi et al., 2004; McMahon et al., 2003; Zhang and Hannink, 2003). Therefore, when tethered to Keap1, Nrf2 is targeted for proteasomal degradation, thereby keeping cellular concentrations low (Itoh et al., 2003; Zhang and Hannink, 2003).

Several groups have identified multiple signaling pathways that may affect Nrf2 activity (Chen and Kong, 2004; Nguyen et al., 2003). The MAPK cascade including ERK2, JNK1 and P38 MAPK is involved in the regulation of $\mathrm{Nrf2}$ activity positively or negatively depends on cell types and inducers. The phosphatidylinositol 3kinase (PI3K) pathway was also known to affect Nrf2 activity and a pharmacological inhibitor of PI3K largely attenuates inducible expression of antioxidative genes in response to $t$-BHQ treatment (Kang et al., 2001; Lee et al., 2001). In addition, phosphorylation of the Ser 40 residue of Nrf2 by protein kinase $\mathrm{C}$ in response to $t$ $\mathrm{BHQ}$ treatment has been directly demonstrated in vivo (Huang et al., 2002). As a upstream event of MAPK cascades for activation of $\mathrm{Nr} 2$, a very recent study by Cho et al. have proposed that members of the $\mathrm{G} \alpha 12$ family involves in regulation of PI3K-dependent $\mathrm{Nr} 2$ activation through modulation of PKC and JNK cascades (Cho et al., 2007). Taken together, these results indicate that Keap1-Nrf2, which is a critical complex for the regulation of the expression of antioxidative genes, is coordinately controlled by diverse, but intersecting, signaling pathways.

Transcriptional regulation of Nrf2 also has been suggested as one of molecular mechanisms to activate the Nif2 pathway. Cho et al. have demonstrated increased transcripts for Nrf2 in mouse lung exposed to hyperoxia. They have identified a point mutation in the promoter region of the nrf2 gene in hyperoxia-sensitive mouse strains and found that this point mutation is associated with differential activation levels of $\mathrm{Nr} 2$ in 
response to hyperoxia (Cho et al., 2002a, b). The ARElike elements were identified in the proximal promoter region of murine nrf2 gene and these elements were shown to mediate the activation of the nrf2 promoter in response to dithiolethione treatment and Nrf2 overexpression in murine keratinocytes (Kwak et al., 2002). Furthermore, a very recent study by Marzec et al. identified a single nucleotide polymorphism (SNP) in the ARE-similar sequences of the human $n r f 2$, and found that this polymorphism is strongly associated with the high risk of development of acute lung injury in human (2007).

\section{GENES REGULATED BY THE NRF2 SIGNALING PATHWAY}

Several groups have performed genome-wide screening using comparisons between wild-type and nrf2-disrupted mice to identify genes regulated by the Keap1Nrf2 pathway. These reports have consistently confirmed that genes promoting the antioxidative capacity of cells are increased by Nrf2 activation. First, Thimmulappa et al. (2002) have identified fifty genes as Nrf2dependent, chemopreventive sulforaphane-inducible genes in the small intestine following daily treatment of sulforaphane for one week. Most of these genes are associated with metabolism of electrophiles and free radicals, and proteins and enzymes enhancing cellular reducing potential such as glucose-6-phosphate dehydrogenase. Second, Kwak et al. (2003b) have reported that expression of three hundred genes was elevated in the liver isolated from D3T-treated wild-type mice and over $80 \%$ of these genes were not induced in nrf2-disrupted mice. These genes can be classified into several categories including typical xenobiotic metabolizing enzymes, antioxidative genes associated with maintaining redox potential, molecular chaperones and $26 \mathrm{~S}$ proteasomes. Antioxidative genes induced by D3T in an Nrf2-dependent manner included thioredoxin, peroxiredoxin and enzymes involved in maintenance of homeostasis of antioxidants, including GSH-biosynthetic enzymes, GSH reductase, and thioredoxin reductase. Major novel gene categories found in this study were molecular chaperones and the $26 \mathrm{~S}$ proteasome. Over $70 \%$ of the subunit genes that collectively form the $26 S$ proteasome were shown to be regulated in an Nif2-dependent manner. Moreover, disruption of the Nrf2 gene abrogated their inducibility. In further studies, it was demonstrated that induction of the catalytic subunit of the 20 S proteasome, PSMB5, was regulated by $\mathrm{Nr} 2$ through interactions with AREs located in its proximal promoter region (Kwak et al., 2003a). These results implicate that possible protective role of the $26 \mathrm{~S}$ proteasome against cytotoxicity mediated by oxidatively damaged proteins. Recently, it has been demonstrated that coordinated induction of the proteasome subunits by sulforaphane resulted in protection against hydrogen peroxide-mediated cytotoxicity and accumulation of oxidized proteins (Kwak et al., 2007).

A third study carried out by Shih et al. (Ishii et al., 2000) has defined the transcription network of Nrf2 in rat brain cells. They focused on identifying genes elevated by Nrf2 itself in neuronal and glial cells from rat embryo by forcing overexpression of Nrf2 protein with a replication-deficient adenovirus. Their results also support the primary role of $\mathrm{Nrf2}$ in the transcriptional activation of ARE-bearing genes such as detoxifying genes and antioxidants. This report, taken together with that of Lee et al. (2003), which used primary cultured cortical astrocytes, strongly suggests a possible neuroprotective role of Nrf2 downstream genes against oxidative/ electrophilic stress.

\section{PHENOTYPES OF NRF2-DISRUPTED MICE}

Based on the different gene expression patterns in the absence of Nrf2, nif2-disrupted mice were hypothesized to be a good model to prove the role of antioxidative defense systems in protection against oxidative damage. Many studies are showing that constitutive expression of several antioxidative genes is reduced in the absence of Nrf2. Levels of transcripts for subunits of GSTA1 and GSTP1, microsomal epoxide hydrolase and ferritin heavy chain were significantly lower in nrf2-disrupted mice than wild-type mice (Kwak et al., 2001a; Ramos-Gomez et al., 2001). Intestinal enzymatic activities for NQO1 and GST were also suppressed $30 \sim 70 \%$ in nrf2-disrupted mice compared to wild-type mice (McMahon et al., 2001). Due to this repressed expression of detoxifying enzymes, nrf2-dirupted mice have been predicted to be more susceptible to toxicities evoked by environmental chemicals and stress. Now there is much data to support this hypothesis. Table 1 summarizes published results examining the susceptibility of nrf2-disrupted mice to oxidative/electrophilic stresses; additional studies are in progress related to aging and neurodegenerative diseases. Several studies have employed chemicals to induce stress. First, administration of the pulmonary toxicant BHT to nrf2-disrupted mice showed a higher mortality than in wild-type mice (Chan and Kan, 1999). Second, nif2-deficient mice were more sensitive to hepatotoxicity induced by acetaminophen intake than wild-type mice (Chan et al., 2001; Enomoto et al., 2001). Third, formation of gastric tumors by benzo[a]pyrene 
Table 1. Phenotypes affected by the nrf2 genotype in chemical exposure and oxidative damage-associated disease models

\begin{tabular}{|c|c|c|c|}
\hline & Type of stressor & Measured phenotype & Reference \\
\hline \multirow[t]{5}{*}{ Chemical exposures } & $\mathrm{BHT}$ & Pulmonary toxicity & Lee et al., 2003 \\
\hline & Acetaminophen & Liver toxicity & Chan et al., 1999, 2001 \\
\hline & Benzo[a]pyrene & Tumor formation in forestomach/ & Ramos-Gomez et al., 2001, 2003 \\
\hline & Diesel exhaust & DNA adduct formation & Aoki et al., 2001 \\
\hline & Aflatoxin $B_{1}$ & DNA adduct formation & Kwak et al., 2001b \\
\hline \multirow[t]{6}{*}{ Disease models } & Bleomycin & Pulmonary fibrosis & Cho et al., 2004 \\
\hline & Hyperoxia & Pulmonary damage & Cho et al., 2002a \\
\hline & Smoking & Emphysema & Rangasamy et al., 2004 \\
\hline & Carageenan & Systemic inflammation & Itoh et al., 2004 \\
\hline & $\begin{array}{l}\text { Autoimmune nephritis in aged } \\
\text { female mice }\end{array}$ & $\begin{array}{l}\text { Nephritis and increased level of autoanti- } \\
\text { bodies }\end{array}$ & Yoh et al., 2001 \\
\hline & $\begin{array}{l}\text { Hemolytic anemia } \\
\text { Systemic autoimmune disease }\end{array}$ & $\begin{array}{l}\text { Hemolysis and increased level of autoan- } \\
\text { tibodies } \\
\text { SLE-like syndrome in multiple organ }\end{array}$ & $\begin{array}{l}\text { Lee et al., } 2004 \\
\text { Li et al., } 2004\end{array}$ \\
\hline
\end{tabular}

treatment was significantly increased in nrf2-disrupted mice compared to wild-type mice (Ramos-Gomez et al., 2001). Fourth, levels of DNA adducts in target tissues are elevated in these knockout mice following exposure to the carcinogens aflatoxin $B_{1}$, benzo[a]pyrene and diesel exhaust (Aoki et al., 2001; Kwak et al., 2001a; Ramos-Gomez et al., 2003). Fifth, indices of lung fibrosis, including hydroxyproline content, collagen accumulation, fibrotic score and cell proliferation, were elevated to a greater extent in bleomycin-treated nrf2disrupted mice than in wild-type mice (Cho et al., 2004).

In addition, phenotypes of nrf2-disrupted mice have been tested using oxidative damage-related disease models (Table 1). First, nrf2-deficient mice were found to be very susceptible to hyperoxic damage of the lung compared to wild-type mice (Cho et al., 2002a). Second, exposure to tobacco smoke, a well-known inducer of oxidative stress, produced emphysema in nrf2-disrupted mice but not in wild-type mice (Rangasamy et al., 2004). Third, Nrf2 has a role in protection from inflammation-induced damage. Carageenan treatment produced significantly higher systemic inflammatory response in the nrf2-disrupted mice than in wild-type mice (Itoh et al., 2004). Fourth, deficiency of Nrf2 is strongly associated with development of autoimmune diseases. Enhanced autoimmunity is also known to be caused by increased protein degradation in response to oxidative damage. Therefore, Nrf2 might have a role in protection against oxidative damage-induced autoimmunity. Targeted disruption of $n$ rf2 leads to development of lupuslike autoimmune nephritis accompanied by higher levels of $\lg G$, IgM, C3, and anti-double strand DNA antibody in female mice over 60 weeks of age (Yoh et al., 2001). Li et al. (2004) have also demonstrated that aged $n r f 2$-deficeint female mice developed multiple organ pathologies resembling human systemic lupus erythe- matosus (SLE) and high levels of autoimmune antibodies were detected in kidney, liver, heart and brain. Also, these mice developed a regenerative immune-mediated hemolytic anemia due to decreased antioxidant potential and increased oxidative stress in erythrocytes (Lee et al., 2004). Taken together, these different phenotypes of nrf2-disrupted mice can be explained by their reduced antioxidant potential and increased oxidative damage in various tissues.

\section{CONCLUDING REMARKS}

Many recent studies demonstrate the pivotal role of $\mathrm{Nrf2}$ in the regulation of antioxidative potential in mammalian cells. Successful development of $n$ rf2-disrupted mice led to new insights into the gene categories regulated by this transcription factor, although descriptions of the Nrf2-regulated networks are far from complete. These mice are also useful in determining critical susceptibility pathways in chemical-mediated toxicities and in the development of murine models of human diseases. Important classes of cancer preventive agents activate the $\mathrm{Nr} 2$ pathway and these models facilitate the identification and molecular study of such compounds. Translation of Nrf2 as a molecular target in humans is underway. Clinical trials using oltipraz in high-risk groups have shown that induction of antioxidative and electrophile detoxication genes by oral administration of oltipraz was an effective regimen to enhance the excretion of the potent human hepatocarcinogen aflatoxin $\mathrm{B}_{1}$ (Wang et al., 1999). It was also demonstrated that anethole dithiolethione (5-( $p$-methoxyphenyl)1,2-dithiole-3-thione) reduced the extent of bronchial dysplasia in smokers in clinical trials, further implicating a role of induction of antioxidative genes in humans (Lam et al., 2002). Current work seeks to identify natu- 
ral products found in foods that activate the Keap1-Nrf2 pathway, as these items may be particularly useful in achieving protection in humans. Clearly, the transcriptional activity of Nrf2 may be a critical determinant in development of toxicity and carcinogenicity following exposure to environmental chemicals.

Modulation of Nrf2 could be also effective in preventing tissue damage, especially in the brain, that is produced by oxidative stress during the process of aging. Decreased transcriptional activity of $\mathrm{Nr} 2$ has been observed in aged rats and this decline appears to play a central role in age-related loss of GSH and a reduced capacity to control cellular homeostasis (Suh et al., 2004). Studies using neuronal cells from animals and humans have highlighted the prospect that Nrf2 controls regulation of antioxidative genes in response to typical enzyme inducers in these cells. Therefore, potential neuroprotective roles of the Nrf2 pathway against aging-associated tissue injuries could be attractive targets. Our continuing challenge will be to identify better inducers - better indirect antioxidative agents - that we can apply in a broad spectrum of chronic disease states.

\section{ACKNOWLEDGEMENTS}

This work was supported by Korean Research Foundation Grant funded by the Korean Government (MOEHRD, Basic Research Promotion Fund) KRF-2006-531E00114.

\section{REFERENCES}

Aoki, Y., Sato, H., Nishimura, N., Takahashi, S., Itoh, K. and Yamamoto, M. (2001). Accelerated DNA adduct formation in the lung of the Nrf2 knockout mouse exposed to diesel exhaust. Toxicol. Appl. Pharmacol., 173, 154-160.

Chan, K., Han, X.D. and Kan, Y.W. (2001). An important function of $\mathrm{Nrf} 2$ in combating oxidative stress: detoxification of acetaminophen. Proc. Natl. Acad. Sci. USA, 98, 4611-4616.

Chan, K. and Kan, Y.W. (1999). Nrf2 is essential for protection against acute pulmonary injury in mice. Proc. Natl. Acad. Sci. USA, 96, 12731-12736.

Chen, C. and Kong, A.N. (2004). Dietary chemopreventive compounds and ARE/EpRE signaling. Free Radic. Biol. Med., 36, 1505-1516.

Cho, H.Y., Jedlicka, A.E., Reddy, S.P., Kensler, T.W., Yamamoto, M., Zhang, L.Y. and Kleeberger, S.R. (2002a). Role of NRF2 in protection against hyperoxic lung injury in mice. Am. J. Respir. Cell Mol. Biol, 26, 175-182.

Cho, H.Y., Jedlicka, A.E., Reddy, S.P., Zhang, L.Y., Kensler, T.W. and Kleeberger, S.R. (2002b). Linkage analysis of susceptibility to hyperoxia. Nrf2 is a candidate gene. Am. J. Respir. Cell Mol. Biol., 26, 42-51.

Cho, H.Y., Reddy, S.P., Yamamoto, M. and Kleeberger, S.R.
(2004). The transcription factor NRF2 protects against pulmonary fibrosis. FASEB J., 18, 1258-1260.

Cho, M.K., Kim, W.D., Ki, S.H., Hwang, J.I., Choi, S., Lee, C.H. and Kim, S.G. (2007). Role of G\{alpha\}12 and G\{alpha\}13 as novel switches for the activity of Nrf2, a key antioxidative transcription factor. Mol. Cell Biol., 27 6195-6208.

Dick, R.A., Kwak, M.K., Sutter, T.R. and Kensler, T.W. (2001). Antioxidative function and substrate specificity of NAD(P)Hdependent alkenal/one oxidoreductase. A new role for leukotriene B4 12- hydroxydehydrogenase/15-oxoprostaglandin 13-reductase. J. Biol. Chem., 276, 40803-40810.

Enomoto, A., Itoh, K., Nagayoshi, E., Haruta, J., Kimura, T., O'Connor, T., Harada, T. and Yamamoto, M. (2001). High sensitivity of Nrf2 knockout mice to acetaminophen hepatotoxicity associated with decreased expression of AREregulated drug metabolizing enzymes and antioxidant genes. Toxicol. Sci., 59, 169-177.

Huang, H.C., Nguyen, T. and Pickett, C.B. (2002). Phosphorylation of $\mathrm{Nrf} 2$ at Ser-40 by protein kinase $\mathrm{C}$ regulates antioxidant response element-mediated transcription. J. Biol. Chem., 277, 42769-42774.

Ishii, T., Itoh, K., Takahashi, S., Sato, H., Yanagawa, T., Katoh, Y., Bannai, S. and Yamamoto, M. (2000). Transcription factor Nrf2 coordinately regulates a group of oxidative stressinducible genes in macrophages. J. Biol. Chem., 275, 16023-16029.

Itoh, K., Chiba, T., Takahashi, S., Ishii, T., Igarashi, K., Katoh, Y., Oyake, T., Hayashi, N., Satoh, K., Hatayama, I., Yamamoto, M. and Nabeshima, Y. (1997). An Nrf2/small Maf heterodimer mediates the induction of phase II detoxifying enzyme genes through antioxidant response elements. Biochem. Biophys. Res. Commun., 236, 313-322.

Itoh, K., Mochizuki, M., Ishii, Y., Ishii, T., Shibata, T., Kawamoto, Y., Kelly, V., Sekizawa, K., Uchida, K. and Yamamoto, M. (2004). Transcription factor Nrf2 regulates inflammation by mediating the effect of 15-deoxy-Delta(12,14)-prostaglandin j(2). Mol. Cell Biol., 24, 36-45

Itoh, K., Wakabayashi, N., Katoh, Y., Ishii, T., Igarashi, K., Engel, J.D. and Yamamoto, M. (1999). Keap1 represses nuclear activation of antioxidant responsive elements by Nrf2 through binding to the amino-terminal Neh2 domain. Genes Dev., 13, 76-86.

Itoh, K., Wakabayashi, N., Katoh, Y., Ishii, T., O'Connor, T. and Yamamoto, M. (2003). Keap1 regulates both cytoplasmic-nuclear shuttling and degradation of Nif2 in response to electrophiles. Genes Cells, 8, 379-391.

Kang, K.W., Cho, M.K., Lee, C.H. and Kim, S.G. (2001). Activation of phosphatidylinositol 3-kinase and Akt by tertbutylhydroquinone is responsible for antioxidant response element-mediated rGSTA2 induction in H4IIE cells. Mol. Pharmacol., 59, 1147-1156.

Kang, K.W., Ryu, J.H. and Kim, S.G. (2000). The essential role of phosphatidylinositol 3-kinase and of p38 mitogenactivated protein kinase activation in the antioxidant response element-mediated rGSTA2 induction by decreased glutathione in H4IIE hepatoma cells. Mol. Pharmacol., 58, 1017-1025

Kensler, T.W. (1997). Chemoprevention by inducers of carcin- 
ogen detoxication enzymes. Environ. Health Perspect, 105, 965-970.

Kobayashi, A., Kang, M.I., Okawa, H., Ohtsuji, M., Zenke, Y., Chiba, T., Igarashi, K. and Yamamoto, M. (2004). Oxidative stress sensor Keap1 functions as an adaptor for Cul3-based E3 ligase to regulate proteasomal degradation of Nrf2. Mol. Cell Biol., 24, 7130-7139.

Kwak, M.K., Cho, J.M., Huang, B., Shin, S. and Kensler, T.W. (2007). Role of increased expression of the proteasome in the protective effects of sulforaphane against hydrogen peroxide-mediated cytotoxicity in murine neuroblastoma cells. Free Radic. Biol. Med., 43, 809-817.

Kwak, M.K., Egner, P.A., Dolan, P.M., Ramos-Gomez, M., Groopman, J.D., Itoh, K., Yamamoto, M. and Kensler, T.W. (2001a). Role of phase 2 enzyme induction in chemoprotection by dithiolethiones. Mutat. Res., 480-481, 305-315.

Kwak, M.K., Itoh, K., Yamamoto, M. and Kensler, T.W. (2002). Enhanced expression of the transcription factor Nif2 by cancer chemopreventive agents: role of antioxidant response element-like sequences in the nrf2 promoter. Mol. Cell Biol., 22, 2883-2892.

Kwak, M.K., Itoh, K., Yamamoto, M., Sutter, T.R. and Kensler, T.W. (2001b). Role of transcription factor Nrf2 in the induction of hepatic phase 2 and antioxidative enzymes in vivo by the cancer chemoprotective agent, $3 \mathrm{H}-1,2$-dimethiole-3-thione. Mol. Med., 7, 135-145.

Kwak, M.K., Ramos-Gomez, M., Wakabayashi, N. and Kensler, T.W. (2004). Chemoprevention by 1,2-dithiole-3-thiones through induction of NQO1 and other phase 2 enzymes. Methods Enzymol., 382, 414-423.

Kwak, M.K., Wakabayashi, N., Greenlaw, J.L., Yamamoto, M. and Kensler, T.W. (2003a). Antioxidants enhance mammalian proteasome expression through the Keap1-Nr2 signaling pathway. Mol. Cell Biol., 23, 8786-8794.

Kwak, M.K., Wakabayashi, N., Itoh, K., Motohashi, H., Yamamoto, M. and Kensler, T.W. (2003b). Modulation of gene expression by cancer chemopreventive dithiolethiones through the Keap1-Nrf2 pathway. Identification of novel gene clusters for cell survival. J. Biol. Chem., 278, 81358145.

Lam, S., MacAulay, C., Le Riche, J.C., Dyachkova, Y., Coldman, A., Guillaud, M., Hawk, E., Christen, M.O. and Gazdar, A.F. (2002). A randomized phase Ilb trial of anethole dithiolethione in smokers with bronchial dysplasia. J. Natl. Cancer. Inst., 94, 1001-1009.

Lee, J.M., Calkins, M.J., Chan, K., Kan, Y.W. and Johnson, J.A. (2003). Identification of the NF-E2-related factor-2dependent genes conferring protection against oxidative stress in primary cortical astrocytes using oligonucleotide microarray analysis. J. Biol. Chem., 278, 12029-12038.

Lee, J.M., Chan, K., Kan, Y.W. and Johnson, J.A. (2004). Targeted disruption of Nrf2 causes regenerative immunemediated hemolytic anemia. Proc. Natl. Acad. Sci. USA, 101, 9751-9756

Lee, J.M., Hanson, J.M., Chu, W.A. and Johnson, J.A. (2001). Phosphatidylinositol 3-kinase, not extracellular signal-regulated kinase, regulates activation of the antioxidant-responsive element in IMR-32 human neuroblastoma cells. J. Biol. Chem., 276, 20011-20016.
Li, J., Stein, T.D. and Johnson, J.A. (2004). Genetic dissection of systemic autoimmune disease in Nrf2-deficient mice. Physiol. Genomics, 18, 261-272.

McMahon, M., Itoh, K., Yamamoto, M., Chanas, S.A., Henderson, C.J., McLellan, L.I., Wolf, C.R., Cavin, C. and Hayes, J.D. (2001). The Cap'n'Collar basic leucine zipper transcription factor Nrf2 (NF-E2 p45-related factor 2) controls both constitutive and inducible expression of intestinal detoxification and glutathione biosynthetic enzymes. Cancer Res., 61, 3299-3307.

McMahon, M., Itoh, K., Yamamoto, M. and Hayes, J.D. (2003). Keap1-dependent proteasomal degradation of transcription factor Nif2 contributes to the negative regulation of antioxidant response element-driven gene expression. $J$. Biol. Chem., 278, 21592-21600.

Nguyen, T., Sherratt, P.J. and Pickett, C.B. (2003). Regulatory mechanisms controlling gene expression mediated by the antioxidant response element. Annu. Rev. Pharmacol. Toxicol., 43, 233-260.

Otieno, M.A., Kensler, T.W. and Guyton, K.Z. (2000). Chemoprotective $3 \mathrm{H}-1,2$-dithiole-3-thione induces antioxidant genes in vivo. Free Radic. Biol. Med., 28, 944-952.

Primiano, T., Gastel, J.A., Kensler, T.W. and Sutter, T.R. (1996). Isolation of CDNAs representing dithiolethione-responsive genes. Carcinogenesis, 17, 2297-2303.

Primiano, T., Li, Y., Kensler, T.W., Trush, M.A. and Sutter, T.R. (1998). Identification of dithiolethione-inducible gene- 1 as a leukotriene B4 12-hydroxydehydrogenase: implications for chemoprevention. Carcinogenesis, 19, 999-1005.

Ramos-Gomez, M., Dolan, P.M., Itoh, K., Yamamoto, M. and Kensler, T.W. (2003). Interactive effects of nrf2 genotype and oltipraz on benzo[a]pyrene-DNA adducts and tumor yield in mice. Carcinogenesis, 24, 461-467.

Ramos-Gomez, M., Kwak, M.K., Dolan, P.M., Itoh, K., Yamamoto, M., Talalay, P. and Kensler, T.W. (2001). Sensitivity to carcinogenesis is increased and chemoprotective efficacy of enzyme inducers is lost in nrf2 transcription factor-deficient mice. Proc. Natl. Acad. Sci. USA, 98, 34103415.

Rangasamy, T., Cho, C.Y., Thimmulappa, R.K., Zhen, L., Srisuma, S.S., Kensler, T.W., Yamamoto, M., Petrache, I., Tuder, R.M. and Biswal, S. (2004). Genetic ablation of Nif2 enhances susceptibility to cigarette smoke-induced emphysema in mice. J. Clin. Invest., 114, 1248-1259.

Suh, J.H., Shenvi, S.V., Dixon, B.M., Liu, H., Jaiswal, A.K., Liu, R.M. and Hagen, T.M. (2004). Decline in transcriptional activity of Nrf2 causes age-related loss of glutathione synthesis, which is reversible with lipoic acid. Proc. Natl. Acad. Sci. USA, 101, 3381-3386.

Talalay, P., De Long, M.J. and Prochaska, H.J. (1988). Identification of a common chemical signal regulating the induction of enzymes that protect against chemical carcinogenesis. Proc. Natl. Acad. Sci. USA, 85, 8261-8265.

Thimmulappa, R.K., Mai, K.H., Srisuma, S., Kensler, T.W., Yamamoto, M. and Biswal, S. (2002). Identification of Nr2regulated genes induced by the chemopreventive agent sulforaphane by oligonucleotide microarray. Cancer Res., 62, 5196-5203.

Venugopal, R. and Jaiswal, A.K. (1996). Nrf1 and Nrf2 posi- 
tively and c-Fos and Fra1 negatively regulate the human antioxidant response element-mediated expression of $\mathrm{NAD}(\mathrm{P}) \mathrm{H}$ :quinone oxidoreductase 1 gene. Proc. Natl. Acad. Sci. USA, 93, 14960-14965.

Venugopal, R. and Jaiswal, A.K. (1998). Nrf2 and Nrf1 in association with Jun proteins regulate antioxidant response element-mediated expression and coordinated induction of genes encoding detoxifying enzymes. Oncogene, 17, 3145-3156.

Wakabayashi, N., Dinkova-Kostova, A.T., Holtzclaw, W.D. Kang, M.L., Kobayashi, A., Yamamoto, M., Kensler, T.W. and Talalay, P. (2004). Protection against electrophile and oxidant stress by induction of the phase 2 response: Fate of cysteines of the Keap1 sensor modified by inducers. Proc. Natl. Acad. Sci. USA, 101, 2040-2045.

Wakabayashi, N., Itoh, K., Wakabayashi, J., Motohashi, H., Noda, S., Takahashi, S., Imakado, S., Kotsuji, T., Otsuka, F., Roop, D.R., Harada, T., Engel, J.D. and Yamamoto, M. (2003). Keap1-null mutation leads to postnatal lethality due to constitutive Nrf2 activation. Nat. Genet., 35, 238245.

Wang, J.S., Shen, X., He, X., Zhu, Y.R., Zhang, B.C., Wang, J.B., Qian, G.S., Kuang, S.Y., Zarba, A., Egner, P.A.,
Jacobson, L.P., Munoz, A., Helzlsouer, K.J., Groopman, J.D. and Kensler, T.W. (1999). Protective alterations in phase 1 and 2 metabolism of aflatoxin B1 by oltipraz in residents of Qidong, People's Republic of China. J. Natl. Cancer Inst., 91, 347-354.

Wasserman, W.W. and Fahl, W.E. (1997). Functional antioxidant responsive elements. Proc. Natl. Acad. Sci. USA, 94 5361-5366

Wild, A.C., Moinova, H.R. and Mulcahy, R.T. (1999). Regulation of gamma-glutamylcysteine synthetase subunit gene expression by the transcription factor Nrf2. J. Biol. Chem., 274, 33627-33636

Yoh, K., Itoh, K., Enomoto, A., Hirayama, A., Yamaguchi, N., Kobayashi, M., Morito, N., Koyama, A., Yamamoto, M. and Takahashi, S. (2001). Nrf2-deficient female mice develop lupus-like autoimmune nephritis. Kidney Int., 60, 13431353.

Zhang, D.D. and Hannink, M. (2003). Distinct cysteine residues in keap1 are required for keap1-dependent ubiquitination of nrf 2 and for stabilization of nrf2 by chemopreventive agents and oxidative stress. Mol. Cell Biol., 23, 81378151 . 